Erratum

\title{
Erratum: Liu et al. Nightlight as a Proxy of Economic Indicators: Fine-Grained GDP Inference around Chinese Mainland via Attention-Augmented CNN from Daytime Satellite Imagery. Remote Sens. 2021, 13, 2067
}

\author{
Haoyu Liu ${ }^{1} \mathbb{D}$, Xianwen He ${ }^{1} \mathbb{D}$, Yanbing Bai ${ }^{1, *}$, Xing Liu ${ }^{2}$, Yilin $\mathrm{Wu}^{1}$, Yanyun Zhao ${ }^{1}$ and Hanfang Yang ${ }^{1}$ \\ 1 Center for Applied Statistics, School of Statistics, Renmin University of China, Beijing 100872, China; \\ 2017201665@ruc.edu.cn (H.L.); 2017201647@ruc.edu.cn (X.H.); wuyilin@ruc.edu.cn (Y.W.); \\ cas-kriu@ruc.edu.cn (Y.Z.); hyang@ruc.edu.cn (H.Y.) \\ 2 Graduate School of Information Sciences, Tohoku University, Sendai 980-8579, Japan; \\ ryu@vision.is.tohoku.ac.jp \\ * Correspondence: ybbai@ruc.edu.cn; Tel.: +86-10-6251-1318
}

check for

updates

Citation: Liu, H.; He, X.; Bai, Y.; Liu, X.; Wu, Y.; Zhao, Y.; Yang, H. Erratum: Liu et al. Nightlight as a Proxy of Economic Indicators: Fine-Grained GDP Inference around Chinese Mainland via Attention-Augmented CNN from Daytime Satellite Imagery. Remote Sens. 2021, 13, 2067. Remote Sens. 2021, 13, 3360. https://doi.org/ $10.3390 /$ rs13173360

Received: 17 June 2021

Accepted: 14 July 2021

Published: 25 August 2021

Publisher's Note: MDPI stays neutral with regard to jurisdictional claims in published maps and institutional affiliations.

Copyright: (c) 2021 by the authors. Licensee MDPI, Basel, Switzerland. This article is an open access article distributed under the terms and conditions of the Creative Commons Attribution (CC BY) license (https:// creativecommons.org/licenses/by/ $4.0 /)$.
The authors wish to make the following corrections to this paper [1].

\section{Incorrect Title}

There is an error in the title. The correct title of the article is "Nightlight as a Proxy of Economic Indicators: Fine-Grained GDP Inference around Chinese Mainland via AttentionAugmented CNN from Daytime Satellite Imagery".

\section{Error in Figures 3 and 5}

In the original article, there was a mistake in Figures 3 and 5 as published. The corrected Figures 3 and 5 appear below. The authors apologize for any inconvenience caused and state that the scientific conclusions are unaffected. The original article has been updated.

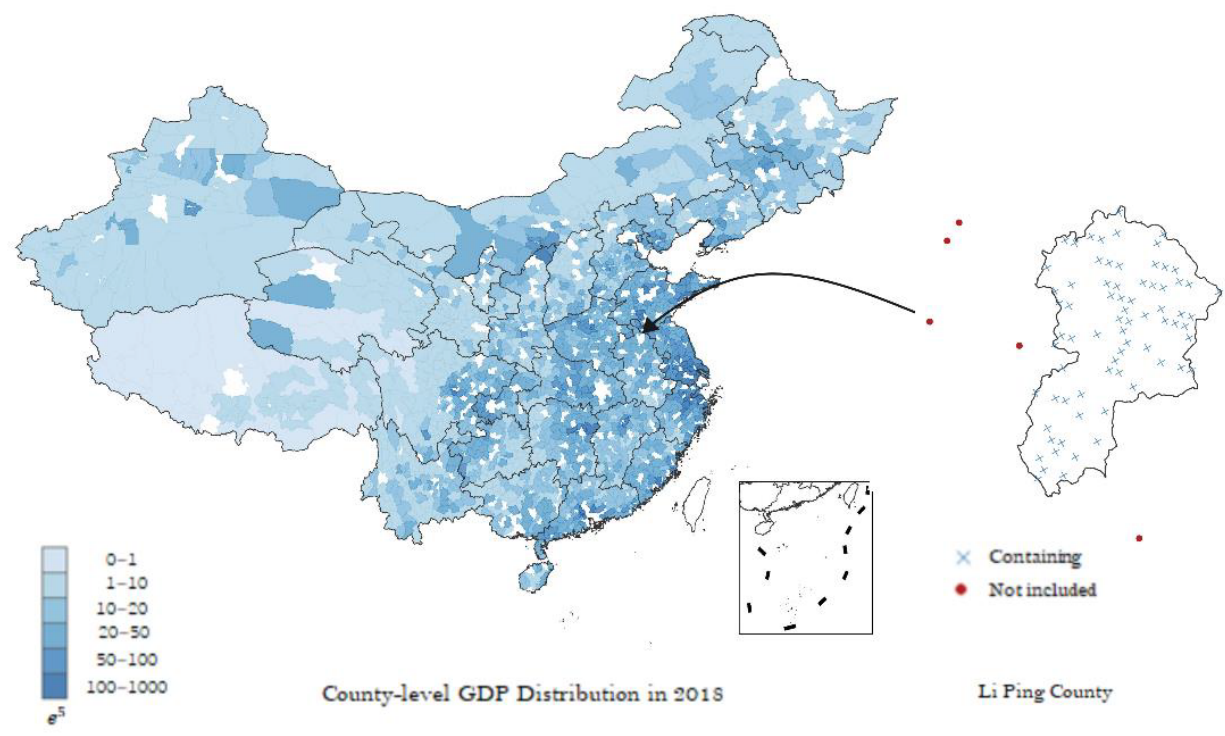

Figure 3. The GDP distribution map of the Chinese Mainland in 2018 (some values along with the boundaries of county-level units are missing), and an example of matching center coordinates and county boundaries. Blue crosses denote center coordinates that fall into the boundary of Liping County, while red points denote centers that do not. 


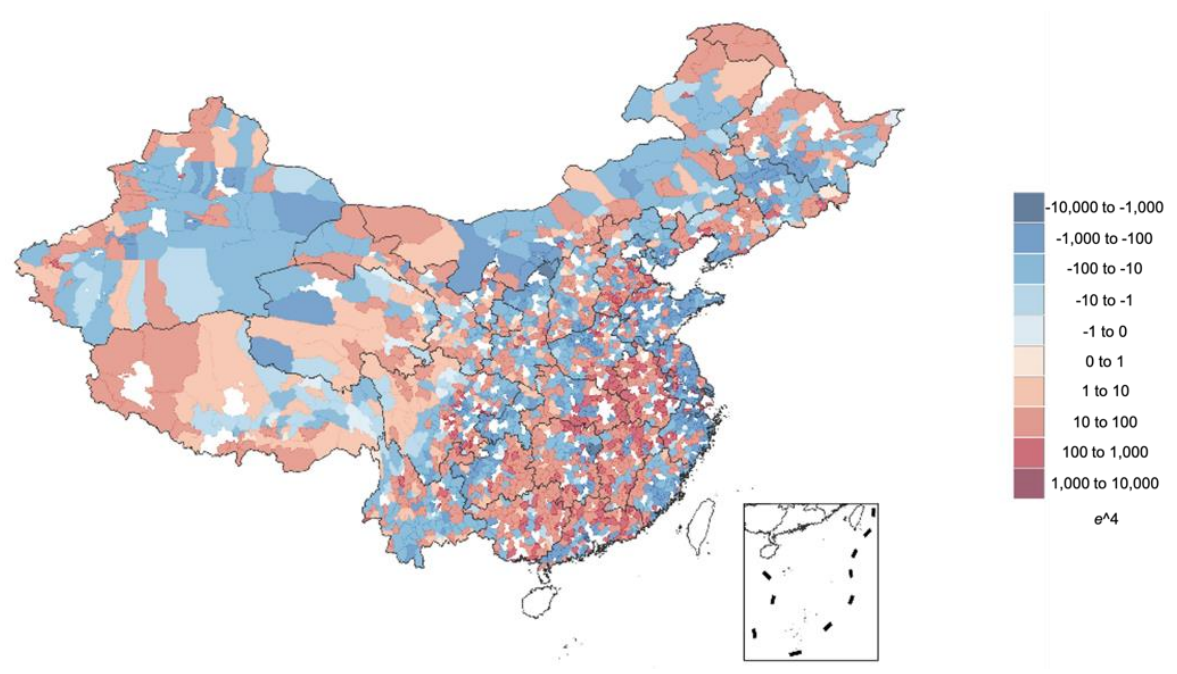

Figure 5. The prediction error map of county-level GDP in 2018. White areas in the map represent regions where data are missing. Due to the large area of the Chinese Mainland, there are a few regions where images are either missing or of poor quality (Hainan Island, for instance). Nevertheless, the number of counties covered by the images we gained is enough for this study.

\section{Incorrect Acknowledgement}

There is an error in the Acknowledgement section. We apologize for this error and state that the scientific conclusions are unaffected. The correct Acknowledgement section of this article is as follows.

Acknowledgement: This work was supported by the Public Computing Cloud, Renmin University of China. We thank Linhao Dong, Ying Hao, Yafeng Wu, Yunhui Xu and Yecheng Tang, students from Renmin University of China; the author gratefully acknowledges the support of the K.C. Wong Education Foundation, Hong Kong.

\section{Reference}

1. Liu, H.; He, X.; Bai, Y.; Liu, X.; Wu, Y.; Zhao, Y.; Yang, H. Nightlight as a Proxy of Economic Indicators: Fine-Grained GDP Inference around Chinese Mainland via Attention-Augmented CNN from Daytime Satellite Imagery. Remote Sens. 2021, $13,2067$. [CrossRef] 